\title{
Adding a neuroanatomical biomarker to an individualized risk calculator for psychosis: A proof-of-concept study
}

\author{
Yoonho Chung a , Jean Addington ${ }^{\mathrm{b}}$, Carrie E. Bearden ${ }^{\mathrm{c}}$, Kristin Cadenhead ${ }^{\mathrm{d}}$, Barbara Cornblatt ${ }^{\mathrm{e}}$, \\ Daniel H. Mathalon ${ }^{\mathrm{f}}$, Thomas McGlashan ${ }^{\mathrm{g}}$, Diana Perkins ${ }^{\mathrm{h}, \mathrm{i}}$, Larry J. Seidman ${ }^{\mathrm{j}, \mathrm{k}}$, Ming Tsuang ${ }^{\mathrm{d}}$, Elaine Walker ${ }^{1}$, \\ Scott W. Woods ${ }^{g}$, Sarah McEwen ${ }^{c}$, Theo G.M. van Erp ${ }^{\mathrm{m}}$, Tyrone D. Cannon ${ }^{\mathrm{a}, \mathrm{g}, *}$, on behalf of the \\ North American Prodrome Longitudinal Study Consortium
}

\footnotetext{
a Department of Psychology, Yale University, 2 Hillhouse Ave., New Haven, CT 06520-8205, United States

${ }^{\mathrm{b}}$ Hotchkiss Brain Institute, Department of Psychiatry, University of Calgary, 3280 Hospital Drive NW, Calgary, AB T2N4Z6, Canada

c Semel Institute for Neuroscience and Human Behavior and Department of Psychology, UCLA, 760 Westwood Plaza, Los Angeles, CA 90095, United States

d Department of Psychiatry, UCSD, 9500 Gilman Drive, La Jolla, CA 92093-0761, United States

e Department of Psychiatry, Zucker Hillside Hospital, 75-59 263rd St., Queens, NY 11004, United States

${ }^{f}$ Department of Psychiatry, UCSF, 401 Parnassus Avenue, San Francisco, CA 94143, United States

g Department of Psychiatry, Yale University, 300 George St., New Haven, CT 06511, United States

h Department of Psychiatry, University of North Carolina, Chapel Hill, 101 Manning Dr, Chapel Hill, NC 27514, United States

${ }^{i}$ Renaissance Computing Institute, University of North Carolina, Chapel Hill, United States

j Department of Psychiatry, Beth Israel Deaconess Medical Center and Harvard Medical School, 401 Park Drive, 2 East, Boston, MA 02215, United States

k Department of Psychiatry, Massachusetts General Hospital and Harvard Medical School, 401 Park Drive, 2 West, Boston, MA 02215, United States

${ }^{1}$ Department of Psychology, Emory University, 487 Psychology Building, 36 Eagle Row, Atlanta, GA 30322, United States

${ }^{\mathrm{m}}$ Department of Psychiatry and Human Behavior, UC Irvine, 101 The City Drive, Irvine, CA 92697, United States
}

\section{A R T I C L E I N F O}

\section{Article history:}

Received 23 July 2018

Received in revised form 27 November 2018

Accepted 19 January 2019

Available online 7 February 2019

Keywords:

Clinical high risk

Risk calculator

Brain age

Structural MRI

Psychosis prodrome

Biomarker
A B S T R A C T

In a recent study, a neuroanatomical-based age prediction model observed neuromaturational deviance among clinical high-risk individuals who developed psychosis. Here we aimed to investigate whether incorporating "brain age gap" (discrepancy between neuroanatomical-based predicted age and chronological age) to the North American Prodromal Longitudinal Study risk calculator would enhance prediction of psychosis conversion. The effect of brain age gap was significant $(\mathrm{HR}=1.21, P=0.047)$, but its predictive variance was found to overlap entirely with age at ascertainment, consistent with the view that greater brain-age gap and earlier age at onset of prodromal symptoms are correlated indicators of insidious-onset forms of psychosis.

\section{Introduction}

We recently published a risk calculator that can ascertain the probability of conversion to psychosis in individual patients with a prodromal risk syndrome (Cannon et al., 2016). Higher levels of unusual thought content and suspiciousness, greater decline in social functioning, lower verbal learning and memory performance, slower speed of processing, and younger age at baseline each contributed to individual risk for psychosis. The multivariate model was comparable in accuracy to risk calculators for cardiovascular disease and cancer and was

* Corresponding author at: Department of Psychology, 2 Hillhouse Avenue, New Haven, CT 06511, United States.

E-mail address: tyrone.cannon@yale.edu (T.D. Cannon). validated in an independent external dataset (Carrión et al., 2016; Kattan et al., 2013; Lee et al., 1999; Specht et al., 2005). Nevertheless, there is room for improvement in the prediction of psychosis. Critically, given the field's current focus on the elucidation of predictive biomarkers, a question of major importance is whether any biological tests can improve prediction over and above the set of clinical, demographic and cognitive measures currently in the risk calculator.

Some promising leads on the use of biological assays to predict psychosis among CHR cases have emerged using empirically-based discovery approaches, including machine learning algorithms for gray matter variations in structural brain images (Koutsouleris et al., 2009, 2014). However, the performance of such models may be limited by difficulty in adequately accounting for the backdrop of variation in normative adolescent brain development and the strong likelihood of heterogeneity in the pathways leading to full psychosis. 
To address these limitations, we recently applied the "brain age" model to individuals diagnosed with psychosis risk syndrome to quantify deviance in brain maturation (i.e. gap between chronological age and "brain age", hereafter referred as brain age gap) for each subject at the point of scan (Chung et al., 2018). The neuroanatomical-based age prediction model was successfully validated in an independent sample. CHR individuals ascertained at younger ages (i.e., 12 to 17 years) were observed to show deviance from the normal neuromaturational pattern (i.e., a gap between "brain age" and chronological age), which in turn was predictive of greater risk of conversion to psychosis and a pattern of stably poor functional outcome. In contrast, individuals who were 18 years of age or older showed age-normative neuroanatomical profiles at ascertainment (i.e., no gap between "brain age" and chronological age). A reevaluation of our prior findings showing a steeper rate of cortical thinning over time among CHR cases who converted to psychosis revealed that this effect was unique to the cases who were 18 years or older at ascertainment and did not apply to the younger cases (Cannon et al., 2015; Chung et al., 2018). This pattern is consistent with the view that neuroanatomical deviance manifesting in early adolescence marks vulnerability to a form of psychosis with an insidious onset and debilitating course of illness, while accelerated cortical thinning marks vulnerability to a more acute onset form of illness that does not manifest until late adolescence and early adulthood.

Here we sought to determine whether the brain age gap effect added significantly to the prediction of psychosis when included in the NAPLS2 risk calculator.

\section{Methods}

This study utilized the NAPLS2 sample, including a total of $476 \mathrm{CHR}$ individuals who were followed up clinically for a minimum of 2 years and who had MRI data available at baseline ( 67 of whom converted to psychosis within a 2-year period). Brain age gap was added to the set of predictors in the NAPLS2 risk calculator: age (years), prodromal symptom severity (modified sum of SIPS items P1:unusual thought content + P2: suspiciousness), symbol coding score from the Brief Assessment of Cognition in Schizophrenia (BACS), score from the Hopkins Verbal Learning Test (sum of trials 1-3; HVLT-R), and decline in social functioning in the year prior to ascertainment (Global Functioning: Social scale); all predictors were selected a priori based on two or more published studies (Cannon et al., 2016), and the risk calculator model was externally validated (Carrión et al., 2016). In order to preserve degrees of freedom for the present analysis, we pruned the model by excluding stressful life events, trauma, and family history as they were not significant predictors in the previous univariate and multivariate analysis (Cannon et al., 2016). Given that brain age score is predictive of psychosis only among younger adolescents who are under 18 years of age (Chung et al., 2018), we computed an interaction (i.e., multiplicative) term between brain gap and bifurcated chronological age, prior to adding to the risk calculator, such that the younger adolescents (aged 12 to 17) were grouped together (and dummy coded as 1), and the older cases (aged 18 and above) were grouped together (dummy coded as 0 ). The pruned base model and the full model that included brain age gap are as summarized below.

Base Model:

Age + SIPS P1\&P2 + Decline in Social Functioning + HVLT + BACS

Full Model (Base Model + Brain Age Gap):

Age + SIPS P1\&P2 + Decline in Social Functioning + HVLT + BACS

+Brain Age Gap (interaction term between brain age gap and dummy coded age)

As in our original study, multivariate Cox proportional hazards regression was used to test the models, which were internally validated using 1000 bootstrap resamples and evaluated with Harrell's C-index to quantify the discrimination ability for separating psychosis converters and non-converters. The C-index is similar in interpretation to the area under the receiver operating characteristic curve, with a range of 0.5 (no discrimination) to 1 (perfect discrimination) and optimized for censored data.

\section{Results}

At the univariate level, the brain age gap effect was predictive of conversion to psychosis (C-index $=0.63 \%, P<0.05$ ) (Chung et al., 2018). The results of the multivariate model testing are shown in Table 1. Based on the bootstrap internal validation, the base model achieved a C-index of 0.70 in this sample. The C-index increased by $1 \%$ when the brain age gap effect was added to the base model, but this effect was not significant. That the brain age gap effect was predictive of conversion to psychosis at the univariate but not multivariate levels indicates that it shares predictive variance in common with other model term (s). Given that earlier age at ascertainment is associated with higher conversion risk and greater brain age gap in this sample, age and brain age gap are likely index the same underlying predictive variance. As shown in Table 1, we tested this hypothesis by removing age from the expanded model; when this was done, the brain age gap effect became significant (Hazard Ratio $=1.21, P=0.047$ ), with no reduction in Cindex compared to the model including age.

\section{Discussion}

The aim of this study was to investigate whether incorporating brain age gap (as an indicator of neuroanatomical deviance from the normal maturational trajectory) to the existing NAPLS2 risk calculator would enhance predictability of conversion to psychosis among individuals diagnosed with psychosis risk syndrome (Cannon et al., 2016). The brain age gap effect was a significant predictor of psychosis at the univariate level (Chung et al., 2018); at the multivariate level, its predictive variance was found to be shared with age at ascertainment, which is to be expected given that earlier age at ascertainment is associated with higher conversion risk and greater brain age gap in this sample and given that earlier age at ascertainment and greater brain age deviation are presumably indicative of insidious onset forms of psychosis.

The contribution of neuroanatomical deviance to the existing psychosis risk prediction model was small. Given that the brain age gap is only relevant for risk prediction in individuals with earlier age at ascertainment (17 years of age or younger), this composite metric is applicable to only about one third of the NAPLS2 sample, as the age

\section{Table 1}

Statistics for individual predictor variables in the multivariate Cox proportional hazards regression analysis of conversion to psychosis.

\begin{tabular}{|c|c|c|c|c|c|c|}
\hline \multirow[t]{2}{*}{ Predictor } & \multicolumn{2}{|c|}{ Base model } & \multicolumn{2}{|c|}{ Full model } & \multicolumn{2}{|c|}{$\begin{array}{l}\text { Full model } \\
\text { without age }\end{array}$} \\
\hline & $\begin{array}{l}\text { Hazard } \\
\text { Ratio }\end{array}$ & $P$ value & $\begin{array}{l}\text { Hazard } \\
\text { Ratio }\end{array}$ & $P$ value & $\begin{array}{l}\text { Hazard } \\
\text { Ratio }\end{array}$ & $P$ value \\
\hline Modified P1 + P2 ${ }^{b}$ & 1.48 & $<0.0001$ & 1.48 & $<0.0001$ & 1.46 & $<0.0001$ \\
\hline $\begin{array}{l}\text { Decline in social } \\
\text { functioning }\end{array}$ & 1.26 & 0.02 & 1.26 & 0.095 & 1.29 & 0.005 \\
\hline HVLT & 0.95 & 0.036 & 0.46 & 0.023 & 0.94 & 0.012 \\
\hline BACS & 0.99 & 0.17 & 0.99 & 0.21 & 0.99 & 0.218 \\
\hline Age & 0.97 & 0.32 & 0.98 & 0.40 & NA & NA \\
\hline Brain age score & NA & NA & 1.18 & 0.08 & 1.21 & 0.047 \\
\hline C-index ${ }^{a}$ & 0.69 & & 0.70 & & 0.70 & \\
\hline
\end{tabular}

BACS = Brief Assessment of Cognition in Schizophrenia; SIPS = Structured Interview for Prodromal Syndromes; HVLT-R = Hopkins Verbal Learning Test-Revised.

a Harrell's C-index (equivalent to the area under the receiver operating characteristic curve) was used to quantify the discrimination ability for separating converters and nonconverters.

$\mathrm{b}$ The base model included only the modified SIPS P1 + P2 score; the C-index for the base model was 0.66 . 
criteria of the psychosis risk syndrome typically extends from 12 to 35 years of age. Therefore, the parameter coefficient for the brain age score is not useful for explaining risk for CHR cases who are 18 years or older at ascertainment. For the older CHR cases who are at heightened risk for late neurodevelopmental disturbances, other biological assays such as proinflammatory cytokine markers from plasma could potentially enhance prediction as it has shown to be closely correlated with steeper rate of cortical thinning over time in older CHR cases (over 18 years of age) who converted to psychosis (Cannon et al., 2015).

Given that CHR individuals with an earlier age at onset of prodromal symptoms with contemporaneous neuroanatomical deficit are still at risk for experiencing later neuromaturational disturbances once they become older adolescents, the maximum of a 2-year follow-up window may not be long enough for younger adolescents to determine their true clinical outcome; this may have introduced false negative cases in terms of clinical outcome if the last assessment point was prior to late adolescence. If this is true, longer follow-ups until their true outcome is realized could potentially improve the relative importance of neuroanatomical deviance as a biological risk factor. In addition, replication of the full model in external validation dataset is warranted to rigorously test generalizability (Cannon et al., 2016; Carrión et al., 2013; Fusar-Poli et al., 2017, 2018).

Although neuroanatomical deviance is presumably a more proximal indicator of insidious onset forms of psychosis than age at ascertainment, given the high cost of a magnetic resonance imaging scan and the fact that it only makes small contribution to the overall risk prediction, measuring brain age is not a practical option for clinical use. However, finding a relatively inexpensive ways to early detect insidious onset types is encouraged as the prognosis for this form of psychosis is poor. Age at prodromal symptom onset, objective records (e.g., medical records, academic reports, psychological evaluations etc.), or interview-based retrospective rating scales such as premorbid adjustment scale (PAS) could be potentially used together for identifying insidious onset types (Cannon-Spoor et al., 1982).

\section{Conflict of interest}

The authors have declared that there are no conflicts of interest in relation to the subject of this study. Dr. Cannon and Dr. Mathalon reports that they are consultants to Boerhinger Ingelheim Pharmaceuticals. Dr. Cannon reports that he is a consultant to Lundbeck A/S.

\section{Funding source}

This work was supported by a collaborative U01 award from the National Institute of Mental Health at the National Institutes of Health (NIH 1R01MH107250-02:S1 to CEB, MH081902 to TDC; MH081857 to BAC; MH081988 to EW; MH081928 to LJS; MH082004 to DP; MH082022 to KC; MH081984 to JA; U01 MH076989 to DHM, MH082022 to SWW) and NIMH P50 MH066286, R01MH107250-S1, and Staglin Music Festival for Mental Health (CEB), NIMH P50 MH080272, and the Commonwealth of Massachusetts (SCDMH82101008006) to L.J.S

\section{Acknowledgements}

The research described in this paper was supported in part by a grant to Yoonho Chung from the American Psychological Association. The authors thank the following individuals for assistance with subject scheduling and/or scan acquisition: Angielette Andaya, Nurit Hirsh and Jamie Zinberg (UCLA); Richard Juelich (BIDMC-Harvard); M. Louis Lauzon, J. Stowkowy and C. Marshall (Calgary); Jason Nunag and Daniel Roman (UCSD); Nicole Popp Santamauro and Hedy Sarofin (Yale).

\section{References}

Cannon, T.D., Chung, Y., He, G., Sun, D., Jacobson, A., van Erp, T.G.M., et al., 2015. Progressive reduction in cortical thickness as psychosis develops: a multisite longitudinal neuroimaging study of youth at elevated clinical risk. Biol. Psychiatry 77 (2), 147-157. https://doi.org/10.1016/j.biopsych.2014.05.023.

Cannon, T.D., Yu, C., Addington, J.M., Bearden, C.E., Cadenhead, K.S., Cornblatt, B.A., et al., 2016. An individualized risk calculator for research in prodromal psychosis. Am. J. Psychiatr. 173 (10), 980-988. https://doi.org/10.1176/appi.ajp.2016.15070890.

Cannon-Spoor, H.E., Potkin, S.G., Wyatt, R.J., 1982. Measurement of premorbid adjustment in chronic schizophrenia. Schizophr. Bull. 8 (3), 470-484.

Carrión, R.E., McLaughlin, D., Goldberg T.E., Auther, A.M., Olsen, R.H., Olvet, D.M., et al., 2013. Prediction of functional outcome in individuals at clinical high risk for psychosis. JAMA Psychiatry 70 (11), 1133-1142. https://doi.org/10.1001/ jamapsychiatry.2013.1909.

Carrión, RE Cornblatt B. A Burton, CZ Tso, IF Auther AM., Adelsheim, S, et al, 2016. Personalized prediction of psychosis: external validation of the NAPLS-2 psychosis risk calculator with the EDIPPP project. Am. J. Psychiatr. 173 (10), 989-996. https://doi.org/10.1176/appi.ajp.2016.15121565.

Chung, Y., Addington, J.M., Bearden, C.E., Cadenhead, K., Cornblatt, B., Mathalon, D.H., et al., 2018. Use of machine learning to determine deviance in neuroanatomical maturity associated with future psychosis in youths at clinically high risk. JAMA Psychiatry 75 (9), 960-968. https://doi.org/10.1001/jamapsychiatry.2018.1543.

Fusar-Poli, P., Rutigliano, G., Stahl, D., Davies, C., Bonoldi, I., Reilly, T., McGuire, P., 2017. Development and validation of a clinically based risk calculator for the transdiagnostic prediction of psychosis. JAMA Psychiatry 74 (5), 493-498. https://doi.org/10.1001/jamapsychiatry.2017.0284.

Fusar-Poli, P., Werbeloff, N., Rutigliano, G., Oliver, D., Davies, C., Stahl, D., et al., 2018. Transdiagnostic risk calculator for the automatic detection of individuals at risk and the prediction of psychosis: second replication in an independent national health service trust. Schizophr. Bull. https://doi.org/10.1093/schbul/sby070.

Kattan, M.W., Yu, C., Stephenson, A.J., Sartor, O., Tombal, B., 2013. Health outcomes research clinicians versus nomogram: predicting future technetium- $99 \mathrm{~m}$ bone scan positivity in patients with rising prostate-specific antigen after radical prostatectomy for prostate cancer. Urology 81 (5), 956-961. https://doi.org/10.1016/j.urology. 2012.12.010.

Koutsouleris, N., Meisenzahl, E.M., Davatzikos, C., Bottlender, R., Frodl, T., Scheuerecker, J., et al., 2009. Use of neuroanatomical pattern classification to identify subjects in atrisk mental states of psychosis and predict disease transition. Arch. Gen. Psychiatry 66 (7), 700-712.

Koutsouleris, N., Riecher-Rössler, A., Meisenzahl, E.M., Smieskova, R., Studerus, E., Kambeitz-Ilankovic, L., et al., 2014. Detecting the psychosis prodrome across highrisk populations using neuroanatomical biomarkers. Schizophr. Bull. https://doi.org/ 10.1093/schbul/sbu078.

Lee, T.H., Marcantonio, E.R., Mangione, C.M., Thomas, E.J., Polanczyk, C.A., Cook, E.F., et al., 1999. Derivation and prospective validation of a simple index for prediction of cardiac risk of major noncardiac surgery. Circulation 100 (10), 1043-1049. https://doi.org/10.1161/01.CIR.100.10.1043.

Specht, M.C., Kattan, M.W., Gonen, M., Fey, J., Van Zee, K.J., 2005. Predicting nonsentinel node status after positive sentinel lymph biopsy for breast cancer: clinicians versus nomogram. Ann. Surg. Oncol. 12 (8), 654-659. https://doi.org/10.1245/ ASO.2005.06.037. 